\section{PSICOLOGÍA IBEROAMERICANA}

Psicología Iberoamericana

ISSN: 1405-0943

psicología.iberoamericana@uia.mx

Universidad Iberoamericana, Ciudad de México

México

Alvarez-Ramírez, Elizabeth; García-Méndez, Mirna; Rivera-Aragón, Sofía Satisfacción marital a través de la dinámica del conflicto y la culpa Psicología Iberoamericana, vol. 23, núm. 1, enero-junio, 2015, pp. 48-57

Universidad Iberoamericana, Ciudad de México

Distrito Federal, México

Disponible en: http://www.redalyc.org/articulo.oa?id=133944230006

- Cómo citar el artículo

Número completo

- Más información del artículo

Página de la revista en redalyc.org

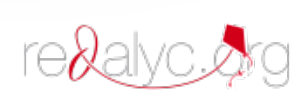

Sistema de Información Científica Red de Revistas Científicas de América Latina, el Caribe, España y Portugal Proyecto académico sin fines de lucro, desarrollado bajo la iniciativa de acceso abierto 


\title{
Satisfacción marital a través de la dinámica del conflicto y la culpa
}

\author{
Marital satisfaction through the dinamic of conflict and blame
}

\author{
Elizabeth Alvarez-Ramírez * \\ Mirna García-Méndez * * \\ Sofía Rivera-Aragón *** \\ Universidad Nacional Autónoma de México
}

\section{RESUMEN}

Con el propósito de establecer si el conflicto y la culpa intervienen en el proceso de la satisfacción marital, 622 participantes de la Ciudad de México, con un rango de edad de 17-60 años $(M=40, S D=10.73)$, respondieron de manera voluntaria la escala de conflicto (Rivera-Aragón, Cruz, Arnaldo \& Díaz-Loving, 2004), el inventario multidimensional de culpa (Alvarez-Ramírez, García-Méndez \& Rivera-Aragón, en prensa) y el inventario multidimensional de satisfacción marital (Cortés, Reyes, Díaz-Loving, Rivera-Aragón \& Monjaraz, 1994). Resultado de una regresión lineal múltiple paso a paso, la satisfacción con la interacción marital es predicha por la reparación del daño $\left(\beta=0.334^{* *}\right)$, conflicto por actividades de la pareja $\left(\beta=-0.203^{\star *}\right)$, chantaje/manipulación $\left(\beta=-0.199^{* *}\right)$, compensación $(\beta=0.134)$, conflicto por la personalidad $\left(\beta=-0.125^{* *}\right)$, conflicto por adicciones $\left(\beta=-0.117^{\star}\right)$ y conflicto por organización/responsabilidad $\left(\beta=-0.115^{*}\right)$.

Palabras clave: Pareja, matrimonio, emociones, reparación, estabilidad marital.

\section{ABSTRACT}

In order to establish whether conflict and blame form part of the process of marital satisfaction, 622 participants from Mexico City, aged between 17 and 60, $(M=40, S D=10.73)$, voluntary took part in the conflict scale (Rivera-Aragón, Cruz, Arnaldo \& Díaz-Loving, 2004), the multidimensional blame inventory of marital satisfaction (Cortés, Reyes, Díaz-Loving, Rivera \& Monjaraz, 1994). The result of a multiple, step-by-step linear regression, satisfaction with marital interaction is predicted by reparation of harm $\left(\beta=0.334^{* *}\right)$, conflict caused by partner's activities $\left(\beta=-0.203^{* *}\right)$, blackmail/manipulation $\left(\beta=-0.199^{* *}\right)$, compensation $(\beta=0.134)$, personality conflict $\left(\beta=-0.125^{* *}\right)$, conflict caused by addictions $\left(\beta=-0.117^{*}\right)$ and conflicts caused by organization/responsibility $\left(\beta=-0.115^{*}\right)$.

Keywords: Couple, marriage, emotions, reparation, marital stability.

Fecha de recepción: 2 de julio de 2014 - Fecha de aceptación: 21 de octubre de 2014

*Licenciada en Psicología. Profesor asignatura “A”, Universidad Nacional Autónoma de México. Teléfono: (55) 52878558. Dirección postal: Edificio de Gobierno planta baja, carrera de psicología, JC Bonilla No. 66 Col. Ejército de Oriente, Iztapalapa, C.P. 09230 México D.F. Correo electrónico psiquecouple@gmail.com

**Doctora en Psicología. Profesor tiempo completo titular “B”, Universidad Nacional Autónoma de México. Dirección postal: Edificio de Gobierno planta baja, carrera de psicología, JC Bonilla No. 66 Col. Ejército de Oriente, Iztapalapa, C.P. 09230 México D.F. Teléfono: (55) 56230648. Correo electrónico: mina@unam.mx

***Doctora en Psicología. Profesor tiempo completo titular "C", Universidad Nacional Autónoma de México. Dirección postal: División de Investigación y Posgrado cubículo "D” edificio "E” o cubículo 7 Mezzanine Facultad de Psicología UNAM. Teléfono: (5255) 56222305 Tel./Fax. (5255) 56222259. Correo electrónico: sofiar@unam.mx 
Durante décadas se ha apreciado la satisfacción marital como un representante del vínculo interpersonal en el matrimonio, en la cual las conductas, afectos y emociones de los integrantes describen al compromiso existente, de manera que se ha considerado como uno de los indicadores de estabilidad y felicidad más importantes en el matrimonio (Becerra-Flores, Roldan \& Flores, 2012). En consecuencia, se piensa que la satisfacción marital está determinada en gran medida por la duración y mantenimiento de la relación, así como su buen funcionamiento, en donde se ven involucrados actitudes y factores tanto positivos como negativos (Neto, 2001). Por esta razón su estudio es importante, porque aporta información sobre la interacción y no sólo sobre la estabilidad y temporalidad de la misma (Armenta-Hurtarte, Sánchez-Aragón \& Díaz-Loving, 2012).

En este contexto existen elementos afectivos como el amor, la comunicación verbal y no verbal manifestados en la relación de pareja; que juegan un papel determinante en la interacción, además de influir en la calidad del matrimonio (Gottman, 1993). Asimismo, estas áreas o factores a los que se enfrentan determinan la dinámica y calidad de su relación, de manera tal que al existir acuerdo entre éstas, la relación se ve fortalecida. De no ser así, la pareja se verá involucrada en interacciones disfuncionales que dificulten el correcto funcionamiento de la interacción, lo que puede generar que la satisfacción marital se vea disminuida (Melero, 2008).

Se ha sugerido que cuando los integrantes de la relación no se sienten satisfechos, tienden a atribuir las conductas positivas de su pareja a causas situacionales, estables y específicas; se obvian por ser evaluadas como algo circunstancial. Al mismo tiempo las conductas negativas se perciben como globales, internas y estables (Bradbury \& Fincham, 1993). En este entorno es muy común culpar al otro de los errores y se magnifican los daños causados, lo que ocasiona un mayor impacto de los sucesos negativos sobre la calidad de la relación, a diferencia de las parejas que viven satisfechas (Fincham, Beach \& Baucom, 1987; Melero, 2008). En muchas ocasiones este tipo de atribuciones y percepciones suelen ser fuente de conflicto en la relación, pues como sugiere Melero (2008) y otros autores, éste surge por diferencias en las necesidades de autonomía o distancia interpersonal, o bien porque uno de los miembros posee un conflicto interno entre sus deseos de autonomía y cercanía.

Evidentemente, el conflicto en la interacción de la pareja es algo inherente, incluso en las relaciones funcionales y equilibradas (Feldman \& Ridley, 2000) debido a que el matrimonio es la unión de dos personas con bagajes educacionales y experiencias diferentes. No obstante, la existencia del conflicto no necesariamente es negativo, en ocasiones, incluso es indicativo de un buen funcionamiento (Markman, 1991). Estas consecuencias benéficas o dañinas se dan en función de cómo se expresa y con qué eficacia es resuelto por los miembros de la relación (Feldman \& Ridley, 2000; Noller \& Fitzpatrick, 1993).

Empero, el impacto negativo de las discrepancias puede depender de dos factores: el grado de satisfacción en la relación en ese momento y el grado de importancia que tenga el tema sobre el que existe el conflicto para cada uno y para la relación (Sanz, 2000). A pesar de considerarse un factor negativo, el conflicto en la interacción de pareja es un proceso donde se busca el equilibrio interno a través de la regulación, con la finalidad de mantener las propiedades con que opera la persona en su relación (Meza, 2010), aunque también se ha encontrado que puede haber diferencia y desacuerdo entre las partes sin generar conflicto. Esto depende de los recursos personales y estructurales para poder definir los límites que establecen los intereses, gustos, necesidades, creencias, etcétera, de cada persona.

La literatura hace referencia a que las fuentes más comunes de conflicto suelen ser las percepciones erróneas o estereotipadas, falta de comunicación, conductas negativas que se repiten, aspecto financiero, crianza de los hijos, relaciones con los parientes, religión, diferencias de valores, expectativas sobre la relación y filosofías de vida (Flores-Galaz, Díaz-Loving \& RiveraAragón, 2002; 2004). Por lo anterior, los conflictos son percibidos como una variable que disminuye la calidad de vida y la satisfacción marital, ya que puede dañar tanto el estado emocional como la integridad de alguno de los miembros.

No obstante, un requerimiento adicional para mantener el matrimonio involucra la habilidad para hacer uso creativo del conflicto: si es manejado destructivamente, la pareja experimentará una relación insatisfac- 
toria; por el contrario, el manejo adecuado incrementara la satisfacción (Rivera-Aragón, Cruz, Arnaldo y Díaz-Loving, 2004). De ahí la importancia atribuida a la solución de conflictos, ya sea que se dé por mediación familiar, negociación o arbitraje, pues de ello dependerá la mejora de la calidad de vida (Ellis y Stuckless, 1996).

Rivera-Aragón (2000) indica que las parejas que no manejan el conflicto con eficiencia ven incrementados con frecuencia sus desacuerdos de forma cada vez más intensa y persistente, a tal grado que los conducen a la dificultad marital e incluso al divorcio. García-Méndez, Rivera-Aragón y Reyes-Lagunes (2008), indican que el compromiso activo, la solución de problemas constructiva, donde prevalece la utilización de estrategias de enfrentamiento positivas, favorecen una alta calidad en la relación marital, pues como menciona Melero (2008) las parejas conflictivas tienden a focalizar más su atención en conductas negativas del otro, desvalorando los sucesos positivos dentro de su relación, lo que funciona como inhibidor de la satisfacción.

Asimismo, Mejía y Laca (2006) mencionan que las mujeres utilizan más que los hombres los mensajes centrados en la pareja, lo que indica una mayor tendencia a evitar los conflictos o a acomodarse a los deseos de su pareja, lo que en parte podría explicarse a partir de parámetros culturales. Consideran además, que esto se debe a las culturas latinas donde las mujeres son educadas socialmente bajo la premisa de rechazo al conflicto, a diferencia de los hombres. En estas circunstancias, los mismos factores suelen generar sentimientos de culpa, principalmente en ellas, pues se les ha creado la ilusión de que son responsables de la felicidad y el bienestar de su familia así como de la relación (Etxebarria \& Pérez, 2003).

En este entorno, se puede observar que el conflicto, así como el descuido de las relaciones cercanas, entre otros, son factores que promueven la experiencia de la culpa (Zabalegui, 1997). Sin embargo su función no sólo es la de causar molestia, sino que se transforma en una emoción autorreflexiva, que promueve autoevaluaciones afectivas negativas de la propia conducta, como resultado de las actitudes de los otros al emitir un juicio sobre las conductas inapropiadas (Abell \& Gecas, 1997). Como resultado se tiende a sentir an- siedad, tristeza, inadecuación, angustia, etcétera. Estas sensaciones motivan la empatía hacia el malestar de la persona víctima del mal proceder, lo que impulsa la búsqueda de la forma adecuada de resarcir el daño causado (Baumeister, Reis \& Delespaul, 1995). Álvarez (1996) considera que existen dos vertientes que motivan las conductas reparadoras: por un lado retribuir el mal causado a otros y restablecer su bienestar emocional; por otro, recuperar la confianza y aceptación social.

En este sentido, autores como Benetti-Mcquoid y Bursik (2005) confirman que la culpa tiene efectos positivos y negativos en las relaciones interpersonales; por ejemplo, arrepentimiento y empatía como consecuencias del daño ocasionado a otros o resultado de conductas consideradas innecesarias que han causado sufrimiento a personas afectivamente cercanas. A este respecto Tangney (1992) propone que el efecto negativo, tiene que ver con cuestiones de control social, juicios morales, por lo que para evitar el malestar, las personas suelen apegarse a las normas establecidas y evitar de nuevo incurrir en la falta. En el contexto de pareja, el efecto positivo se hace evidente al inducir conductas dirigidas a resarcir el daño, con la finalidad de restaurar el bienestar del compañero, tales como pedir perdón, autocorregirse, retribuir y reparar el daño (Etxebarria, Pascual \& Conejero, 2010; Hoffman, 2002).

En este entorno, si en el matrimonio hay situaciones conflictivas que afecten la estabilidad de la díada, existe la posibilidad de que la relación mejore si los miembros de la relación son empáticos y reparan el daño causado a su pareja, debido a que estas acciones generan bienestar lo que potencializará la satisfacción de los cónyuges. Esto se explica a partir de la función conciliadora de la culpa (Baumeister, Stillwell \& Heartherton, 1994), razón por la que se considera un proceso en la mejora de las relaciones interpersonales, como explican algunos autores (Etxebarria et al., 2010; Hoffman, 2002).

Como se puede apreciar a través de la literatura expuesta, la satisfacción marital es un constructo que juega un papel muy importante en la estabilidad y duración de las relaciones, por lo que es de suma importancia conocer los procesos que la afectan, así como los que promueven su mejora. Con base en lo propuesto 
con anterioridad el objetivo de este estudio fue conocer los factores del conflicto y la culpa que predicen la satisfacción con la interacción en la relación de pareja.

\section{MÉTODO}

\section{Participantes}

Participaron 622 personas: 316 hombres y 306 mujeres de la Ciudad de México, con un rango de edad de 1760 años $(M=40, S D=10.73)$. El nivel de estudios fue de primaria a posgrado. En lo que compete al estado civil, el $67.5 \%$ fueron casados y el $32.5 \%$ vivían en unión libre.

\section{Instrumentos}

Se utilizó el inventario multidimensional de culpa en la relación de pareja (Alvarez-Ramírez, García-Méndez y Rivera-Aragón, en prensa) que consta de cinco subescalas que miden la culpa en la relación de pareja: significado de culpa (varianza $=51.45 \% \alpha=0.842$ ), causas de la culpa (varianza $=63.13 \% \alpha=0.947$ ), consecuencias (varianza $=58.91 \% \alpha=0.861$ ), revaloración (varianza $=$ $60.63 \% \alpha=0.828$ ) y efectos de la culpa que a su vez consta de tres factores: reparación del daño, chantajemanipulación y compensación (varianza $=52.66 \%$ $\alpha=0.807$ ). Para los fines de este estudio sólo se utilizó la subescala efectos de la culpa.

También se usó la escala de conflicto (Rivera-Aragón, Cruz, Arnaldo \& Díaz-Loving, 2004) que consta de 66 reactivos distribuidos en 14 factores que explican el $66.10 \%$ de la varianza con un alpha de Cronbach global de 0.86. Los factores son: educación de los hijos, ideología/intereses, actividades de la pareja, actividades propias, desconfianza, relaciones con la familia política, religión, personalidad, sexualidad, dinero/ irresponsabilidad, celos, adicciones, orden /responsabilidad, y familia de origen.

El inventario multifacético de satisfacción marital (IMSM) (Cortés, Reyes, Díaz-Loving, Rivera-Aragón \& Monjaraz, 1994) contiene 48 reactivos, con una escala de respuesta tipo Likert con cinco opciones de respuesta, que van de 1 (me disgusta mucho) a 5 (me gusta mucho) distribuidos en seis factores: interacción conyugal, físico-sexual, organización-funcionalidad, familia, diversión, hijos. Tiene un alpha de 0.85. Para los fines de este estudio se trabajó sólo con el factor interacción de este instrumento.

\section{Procedimiento}

Se contactó a personas en centros de trabajo y hogares y se les explicó brevemente el objetivo de la investigación. A quienes accedieron a participar de manera voluntaria se les entregaron los instrumentos pertinentes. Se hizo énfasis en que la información sería anónima y confidencial, además de obtener el consentimiento informado. Después se procedió a generar la base de datos en el programa SPSS21 para realizar el análisis de regresión lineal múltiple paso a paso.

\section{RESULTADOS}

Para lograr el objetivo del presente estudio se realizó en primer lugar una correlación producto momento de Pearson con la finalidad de establecer la relación entre el conflicto, los efectos de la culpa, y la satisfacción marital. Los resultados indican la existencia de correlaciones significativas y en su mayoría negativas. Éstas se pueden observar en la Tabla 1.

Respecto de los factores que corresponden a los efectos de la culpa, las correlaciones son significativas: las más altas se aprecian en el aspecto positivo como la reparación del daño: a mayor empatía y búsqueda del bienestar de la pareja, se experimentará mayor satisfacción. Por su parte chantaje/manipulación correlacionó de manera negativa. Este dato alude que aliviar los sentimientos de culpa a través de estrategias de manipulación, así como la compensación y la falta de interés en bienestar del cónyuge, generan malestar y disminuyen la estabilidad y satisfacción en la interacción marital.

Posteriormente se realizó un análisis de regresión lineal paso a paso, en el cual las variables independientes fueron los factores de la escala de conflicto: educación de los hijos, ideología/intereses, actividades de la pareja, actividades propias, desconfianza, relaciones con la familia política, religión, personalidad, sexualidad, dinero/irresponsabilidad, celos, adicciones, orden/responsabilidad, y familia de origen. Los efectos positivos de la culpa fueron la reparación del daño, el chantaje/manipulación y la compensación. La variable dependiente fue el factor interacción de la escala de satisfacción marital. 
Tabla 1. Correlación de Pearson entre el conflicto, la culpa y la satisfacción con la interacción marital

\begin{tabular}{|c|c|c|c|}
\hline & Interacción & $M$ & DS \\
\hline Educación de los hijos & $-0.400 * *$ & 3.73 & 0.84 \\
\hline Ideología/intereses & $-0.434^{* *}$ & 3.52 & 0.81 \\
\hline Actividades de la pareja & $-0.432 * *$ & 3.74 & 1.01 \\
\hline Actividades propias & $-0.320 * *$ & 3.77 & 0.93 \\
\hline Desconfianza & $-0.391 * *$ & 3.80 & 0.77 \\
\hline Familia política & $-0.315^{* *}$ & 3.84 & 0.83 \\
\hline Religión & $-0.199 * *$ & 4.43 & 0.94 \\
\hline Personalidad & $-0.429 * *$ & 3.44 & 0.86 \\
\hline Sexualidad & $-0.317^{* *}$ & 3.81 & 0.99 \\
\hline Dinero/irresponsabilidad & $-0.322 * *$ & 3.76 & 0.82 \\
\hline Celos & $-0.183^{* *}$ & 3.99 & 0.91 \\
\hline Adicciones & $-0.196 * *$ & 4.14 & 0.81 \\
\hline Orden/responsabilidad & $-0.413 * *$ & 3.92 & 0.84 \\
\hline Familia de origen & $-0.227^{* *}$ & 4.16 & 0.86 \\
\hline Reparación del daño & $0.529 * *$ & 4.71 & 0.97 \\
\hline Chantaje/manipulación & $-0.322 * *$ & 2.49 & 1.07 \\
\hline Compensación & $-0.228 * *$ & 3.35 & 1.14 \\
\hline M & 3.89 & & \\
\hline DS & 0.90 & & \\
\hline
\end{tabular}

Nota: ${ }^{*} \mathrm{p}<.01,{ }^{*} \mathrm{p}<.05$

Como resultado del análisis se creó un modelo donde la reparación del daño se incorporó a la ecuación como el primer predictor; explicó el 28\% de varianza $(F(1,620)=241.913, p<.001)$. En el segundo paso, el conflicto por las actividades de la pareja, explicaron el 39\% de la varianza; el cambio en $R^{2}=0.105, F(1,619)=193.701, p<.001$. En el tercer paso, el chantaje/manipulación, explicó el $40 \%$ de varianza y hubo un cambio en $R^{2}=0.015, F(1,618)=$ $137.185, p<.001$. En el cuarto paso, la compensación explicó el $42 \%$ de varianza y hubo un cambio en $R^{2}=$ $0.015, F(1,617)=109.45, p<.001$. En el quinto paso, el conflicto por la personalidad, explicó el $43 \%$ de varianza, con un cambio en $R^{2}=0.011, F(1,616)=$ $91.48, p<.001$. En el sexto paso, el conflicto por adicciones, explicó el $43 \%$ de varianza, el cambio fue en $R_{2}=0.005, F(1,615)=77.77, p<.005$. En el séptimo paso, el conflicto por el orden y la responsabilidad, explicó el $44 \%$ de varianza, con cambio en $R_{2}=0.006$ $F(1,614)=68.20, p>.005$. 
Figura 1. Modelo predictivo de la satisfacción con la interacción marital.

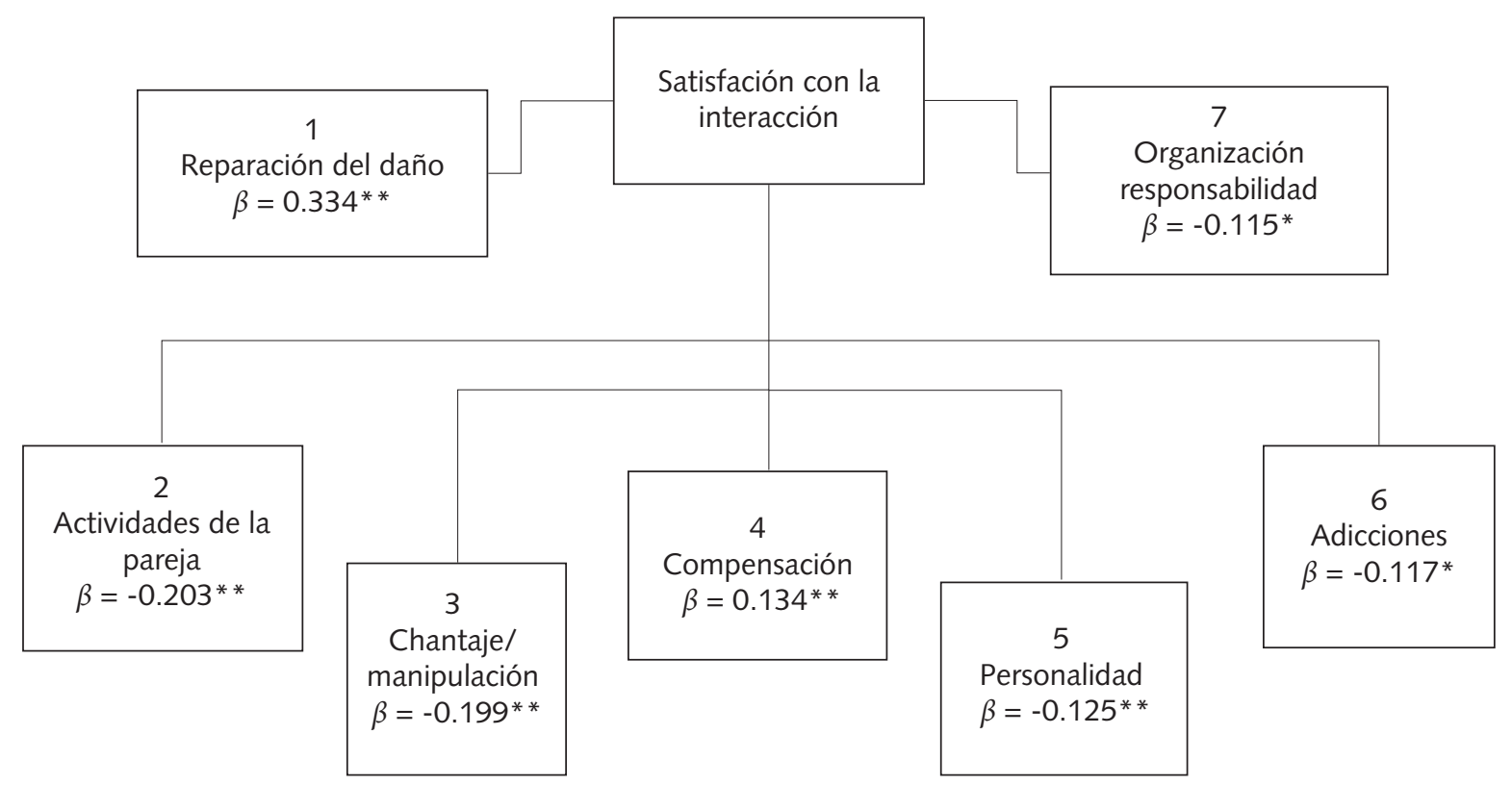

El modelo y las betas correspondientes a cada predictor generado en el séptimo modelo se reportan en la figura 1. Como se puede apreciar en los datos, para que los integrantes de la pareja tengan una interacción satisfactoria necesitan en primer lugar hacer uso de los efectos positivos de la culpa como la reparación del daño, eludir el conflicto por las actividades externas de la pareja, evitar hacer uso del chantaje o manipulación, impedir compensar a la pareja para dejar de sentir culpa, no generar disputas con respecto de la personalidad del otro, así como por las adicciones existentes y por la falta de organización y responsabilidad dentro de la dinámica diaria en la relación.

\section{DISCUSIÓN}

Como se ha escrito, la satisfacción marital es un indicador de estabilidad y felicidad, por lo cual su estudio representa una labor importante, pues de la satisfacción que las personas manifiesten sentir con su relación dependerá su funcionamiento y duración (Becerra-Flores et al., 2012). La interacción conyugal es una de las dimensiones más importantes de la satisfacción porque se involucran aspectos emocionales, afectivos y de comprensión que facilitan la interacción de la pareja (Cortés et al., 1994). Los resultados obtenidos refieren que las variables de estudio mantienen una relación significativa, en las cuales a través de la dinámica entre el conflicto y los efectos de la culpa, la pareja experimenta la satisfacción con su interacción y con su cónyuge.

Respecto del conflicto, este estudio corroboró lo propuesto en estudios anteriores (Flores-Galaz et al., 2002; 2004; Meza, 2010; Sanz, 2000) respecto de su efecto negativo en la interacción de pareja, lo cual lleva a la disminución de la satisfacción marital. Esta incidencia se observa principalmente cuando el conflicto tiene que ver con situaciones como la diferencia de ideologías, los intereses y la personalidad, las actividades que tiene la pareja que propician menos tiempo compartido por los cónyuges, así como la responsabilidad o la falta de ella en actividades cotidianas que repercuten en la dinámica familiar. En las discrepancias, la calidad de vida así como la satisfacción disminuyen y a su vez afectan la estabilidad emocional de uno o ambos integrantes de la relación.

Respecto de la culpa, entendida como una emoción de origen negativo (Álvarez, 1996; BenettiMcquoid \& Bursik, 2005; Etxebarria, 2006), en este 
estudio en particular se puso especial atención en sus efectos, que diversos autores reportan como positivos pues llevan a la retribución del daño y la mejora de las relaciones interpersonales (Etxebarria et al., 2010; Hoffman, 2002; Zabalegui, 1997). Se pudieron advertir correlaciones importantes en sus tres dimensiones. En las negativas como la compensación y el chantaje/ manipulación se muestra un efecto negativo sobre la satisfacción marital, lo que indica que si las personas tienden a buscar aliviar la culpa a través del chantaje o la manipulación; es decir, a ejercer poder sobre el otro para obligar, chantajear o persuadirlo con la finalidad de que asuma la responsabilidad de los conflictos, no resuelve la situación conflictiva; por el contrario, impactará directamente en el bienestar de los cónyuges y su satisfacción.

Del mismo modo, la propensión a dar obsequios, comprar flores, invitar a la pareja a salir, entre otras acciones con la finalidad de aliviar la sensación de malestar que causa la culpa, tampoco favorece la satisfacción con la interacción en la pareja, debido a que al final no se afronta la situación real: las personas sólo recurren a ella para sentirse mejor sin considerar la importancia de restaurar el bienestar emocional y psicológico de su pareja, lo cual conduce en dirección opuesta a la satisfacción o felicidad con la relación.

Respecto de la reparación del daño se obtuvieron relaciones positivas pues, como lo mencionaron en estudios anteriores (Baumeister et al., 1994; Etxebarria et al., 2010; Hoffman, 2002), el objetivo principal es resarcir el daño y generar una sensación de bienestar y mejora en los otros, principalmente en las relaciones interpersonales. Los hallazgos corroboran lo descrito y sugieren que si las personas, a través de la autorreflexión, se atribuyen la responsabilidad de la conducta disruptiva o dañina y evitan volver a realizarla, así como mostrar empatía, amor y además piden perdón a su pareja, se restablece el equilibrio emocional y será más satisfactoria la interacción entre ellos.

Con relación al objetivo de estudio que planteaba conocer los factores del conflicto y los efectos de la culpa que predijeran la satisfacción con la interacción de pareja, los resultados mostraron un modelo que confirma la existencia de correlaciones significativas entre las variables de estudio, en el cual se muestran efectos negativos del conflicto, el chantaje/manipulación y los positivos de la reparación del daño.

Los datos obtenidos refieren que para que los integrantes de la relación se sientan satisfechos con su interacción y su pareja, en primer lugar necesitan hacer uso de la reparación del daño, pues a pesar de que el conflicto es inevitable en la interacción de los cónyuges siempre habrá la oportunidad de restablecer el bienestar de los mismos al emplear la función conciliadora y reparadora de la culpa, lo que coincide con estudios anteriores en los cuales los teóricos reportan que incrementar la empatía, amor y la capacidad de disculparse con el otro favorece relaciones más satisfactorias y felices (Baumeister et al., 1994; Etxebarria \& Apodaca, 2008).

En este proceso, el conflicto generado por las actividades de la pareja afecta de manera negativa a la satisfacción; es decir, el hecho de que el cónyuge tenga diversas actividades fuera de casa y dedique menos tiempo a la relación y a la pareja, causa conflicto en la dinámica diaria. En consecuencia, las horas de tiempo compartido disminuyen y la interacción tiende a tornarse insatisfactoria. Sin embargo, la tendencia indica que si se disminuyen las discusiones por estas circunstancias la relación marital será más satisfactoria. Debido a que el conflicto es inherente en la relación de pareja, es indispensable un adecuado manejo de éste, como ya lo sugerían Rivera-Aragón et al. (2004), pues de ello dependerá el bienestar de los integrantes de la relación y la calidad de ésta.

En contraste, el uso de del chantaje y la manipulación aparece como factor importante en el proceso de predicción de la satisfacción. Cuando los individuos causan un daño a su pareja de manera directa o indirecta por medio de una conducta o actitud, y deciden evitar el malestar de la culpa al manipular o chantajear al otro con la finalidad de que se atribuya la culpa de lo sucedido o lo olvide, habrá un efecto negativo. Como lo explican Rivera-Aragón y Díaz Loving (2002) usar estas estrategias de poder negativas favorece el conflicto e incrementa la insatisfacción.

Del mismo modo, cuando los causantes del daño lo compensan a través de regalos, que pueden ser costosos, para que el otro deje de sentirse molesto o triste, o bien con la finalidad de evitar su propio malestar, se produce un efecto negativo pues la pareja no recupera 
su estabilidad emocional. Si esta situación por el contrario, como proponen Benetti-Mcquoid y Bursik (2005), se afronta al manifestar empatía hacia el otro y de este modo preocuparse por los efectos y la corrección de las propias acciones (Hoffman, 2002), genera un bienestar emocional en ambos, lo que se traduce en este contexto en un incremento en la satisfacción marital expresada.

Otros factores que intervienen como predictores de una interacción satisfactoria se reflejan en el conflicto generado por la diferencia de personalidades y por la existencia de adicciones en uno o ambos miembros. La falta de orden y responsabilidad suelen ser fuentes constantes de conflicto en la relación (FloresGalaz et al., 2004). Esto propone la importancia de recurrir al dialogo, la comprensión y aceptación de su pareja a través de la búsqueda de un proceso de adaptación para establecer una interacción estable, así como un adecuado manejo del conflicto para mejorar la calidad en la relación. Algunos autores mencionan que a pesar de que el conflicto generado por este tipo de situaciones no sea relevante, cuando existe nivel de satisfacción bajo se contribuye a potencializarlo y atribuir en mayor medida la responsabilidad al cónyuge (Bradbury \& Fincham, 1993; Fincham et al., 1987; Melero, 2008), en un movimiento cíclico que cada vez genera mayor malestar entre los integrantes de la díada y disminuye, por tanto, la satisfacción. Por consiguiente, la importancia atribuida a la solución de conflictos, por mediación familiar, negociación o arbitraje, es fundamental pues de ello dependerá la mejora de la calidad de vida (Ellis \& Stuckless, 1996).
En conclusión, la relación entre el conflicto, los efectos de la culpa y la satisfacción con la interacción marital permiten reflexionar sobre la importancia de esta emoción en la interacción de la pareja. Como plantean algunos autores (Baumeister et al., 1994; Etxebarria et al., 2008) la culpa genera otras emociones negativas que motivan a las personas a acercarse a su pareja y realizar o evitar conductas generadoras de daño, porque a pesar de su origen negativo, su función reparadora y promotora de la empatía tiene un efecto significativamente positivo en la satisfacción que ambos cónyuges experimentan en la relación.

Los datos reportados posibilitaron la confirmación de lo propuesto en la literatura de la satisfacción marital, además en coincidencia con Armenta-Hurtarte et al. (2012) permiten conocer la dinámica y las variables que intervienen en el proceso y la importancia de una interacción satisfactoria para el buen desarrollo y mantenimiento de las relaciones maritales.

Evidentemente existen factores negativos que afectan la interacción, no obstante, otros positivos, como los efectos de la culpa, la favorecen. Los resultados aquí reportados, además, permiten concluir que la inherencia del conflicto y la culpa en la relación de pareja no implica relaciones negativas y/o destinadas al fracaso. Por el contrario, los teóricos o terapeutas pueden acceder a esta información y considerar hacer uso creativo de ella, al aplicar estrategias con la finalidad de favorecer o fortalecer, en individuos y parejas, habilidades para adquirir tácticas de enfrentamiento y manejo de estas adversidades y, por ende, experimentar estabilidad y satisfacción marital. • 


\section{REFERENCIAS}

Abell, E. \& Gecas, V. (1997). "Guilt, shame, and family socialization: a retrospective study." Journal of $\mathrm{Fa}$ mily Issues, 18(2), 99-123.

Álvarez, G. N. (1996). La tendencia a sufrir el castigo: una contribución a la teoría de la culpa. Alcalá Henares, País: Universidad de Alcalá de Henares.

Armenta Hurtarte, C. \& Díaz-Loving, R. (2008). “Comunicación y satisfacción: analizando la interacción de pareja.” Psicología Iberoamericana, 16(1) 23-27. En: http://www.redalyc.org/articulo.oa?id= 133915922004

Baumeister, R., Reis, H. \& Delespaul, P. (1995). "Subjective and experimental correlates of guilt in daily life." Personality and social Psychology Bulletin, 21(12), 1256-1268.

Baumeister, R., Stillwell, A. \& Heartherton, T. (1994) Guilt: an interpersonal approach. Psychological Bulletin, 115(2), 243-267.

Becerra-Flores, S., Roldan, W. \& Flores W. (2012). "Factores de enriquecimiento, mantenimiento, comunicación y bienestar en la relación marital." Revista de Psicología GEPU, 3(1), 37-62.

Benetti-McQuoid, J. \& Bursik, K. (2005). "Individual differences in experiences of and responses to guilt and shame: examining the lenses of gender and gender role." Sex Roles, 53(1, 2), 133-142.

Bradbury, T. N. \& Fincham, E. D. (1993). "Assessing dysfunctional cognition in marriage: a reconsideration of the relationship belief inventory." Psychological Assessment, 5, 92-101.

Cortés, S., Reyes, D., Díaz-Loving, R., Rivera-Aragón, S. \& Monjaraz, J. (1994). "Elaboración y análisis psicométrico del Inventario Multifacético de Satisfacción Marital (IMSM)." La Psicología Social en México, V, 123-130.

Ellis, D. \& Stuckless, N. (1996). Mediating and negotiating marital conflicts. Thousand Oaks, CA: Sage.

Etxebarria, I. (2000). “Guilt: an emotion under suspicion.” Psicothema, 12(11), 101-108.

Etxebarria, I. (2006). Los sentimientos de culpa: ¿qué hacemos con ellos? Málaga, País: Arguval.

Etxebarria, I. \& Apodaca, P. (2008). "Both Freud and Hoffman are right: anxious-aggressive and empathic dimensions of guilt." The Spanish Journal of Psychology, 11(1), 159-171.
Etxebarria, I., Pascual, A. \& Conejero, S. (2010) “PPuede considerarse la culpa un índice de sensibilidad interpersonal? Reactividad interpersonal y culpa interpersonal." Ansiedad y estrés, 16(2-3), 201-214.

Etxebarria, I. \& Pérez, J. (2003). “¿Qué nos hace sentir culpa? Categorías de eventos en adolescentes y adultos de uno y otro sexo." Estudios de Psicología, 24 (2), 241-252.

Feldman, C. M. \& Ridley, C. A. (2000). "The role of conflict-based communication responses and outcomes in male domestic violence toward female partners." Journal of Social and Personal Relationships, 17, 552-573.

Fincham, F. D., Beach, S. R. \& Baucom, D. H. (1987). "Attribution processes in distressed and nondistressed couples." Journal of Personality and Social Psychology, 52, 739-748.

Flores-Galaz, M. M., Díaz-Loving, R. \& Rivera-Aragón S. (2002). "El paso del tiempo, los hijos y el ingreso en la relación con la satisfacción marital." Revista de Psicología Social y Personalidad, 18(1) 37-54.

Flores-Galaz, M. M., Díaz-Loving, R. \& Rivera-Aragón S. (2004). "Validación psicométrica del inventario de negociación del conflicto en parejas de una subcultura tradicional.“ $R I D E P, 17(1), 39-55$.

García-Méndez, M., Rivera-Aragón S. \& Reyes-Lagunes, I. (2008). "El conflicto y las estrategias de enfrentamiento empleadas en la relación marital." Revista Mexicana de Psicología, número especial, 120-130.

Ellis, D. \& Stuckless, N. (1996). Mediating and negotiating marital conflicts. Thousand Oaks, CA: Sage.

Gottman, J. (1993). "A theory of marital dissolution and stability." Journal of Family Psychology, 7, 57-75.

Hoffman, M. L. (2002). Desarrollo moral y empatía: implicaciones para la atención y la justicia. Barcelona, España: Idea Books.

Markman, H. J. (1991). "Constructive conflicts is not an oxymoron." Behavioral assessment, 13, 83-96.

Mejía, C. J. y Laca, A. F. (2006). “Estilos de comunicación en el conflicto y confianza en las propias decisiones." Enseñanza e investigación en psicología, 11(2), 347-358.

Melero, R. (2008). La relación de pareja. Apego, dinámicas de interacción y actitudes amorosas: consecuencias so- 
bre la calidad en las relaciones (tesis doctoral inédita.) Universidad de Valencia, Valencia, ES.

Meza, M. (2010). "Estereotipos de violencia en el conflicto de pareja: construcciones y prácticas en una comunidad mexicana" (tesis doctoral inédita.) Universidad Nacional Autónoma de Barcelona, Barcelona.

Neto, F. (2001). "Love styles of three generations of women." Marriage and Family Review, 33(4), 19-30.

Noller, P. \& Fitzpatrick, M. A. (1993). Communication in family relationships. New York: Prentice-Hall.

Rivera-Aragón, S. (2000). “Conceptualización, medición y correlatos de poder y pareja: una aproximación etnopsicológica." (Tesis doctoral inédita.) Facultad de Psicología de la Universidad Nacional Autónoma de México, México.
Rivera-Aragón, S., Cruz del Castillo, C., Arnaldo O. y Díaz-Loving, R. (2004). "Midiendo el conflicto en la pareja." La Psicología en México, AMEPSO, X, 229-236.

Rivera-Aragón, S. y Díaz-Loving, R. (2002). La cultura del poder en la pareja. México: Porrúa-UNAM.

Tangney, J. P. (1992) Situational determinants of shame and guilt in young adulthood. Personality and Social Psychology Bulletin, 18, 199-206.

Sanz, A. (2000). La vida en pareja. Cómo resolver las dificultades de la comunicación. Madrid, España: Temas de hoy.

Zabalegui, L. (1997) ¿Por qué me culpabilizo tanto? Bilboa, País: Desclée Brouwer. 